\title{
Nuevos abordajes en la epiduroscopia para el síndrome de cirugía lumbar fallida. Nuevas oportunidades
}

\author{
D. Abejón González \\ Director Departamento de Unidad del Dolor. Hospital Universitario Quirón. Madrid. Profesor asociado de la \\ Universidad Europea de Madrid, España
}

Martín Avellanal, Gonzalo Diaz-Reganon, Alejandro Orts, Lucio Gonzalez-Montero and Irene Riquelme. Transforaminal Epiduroscopy in Patients with Failed Back Surgery Syndrome. Pain Physician 2019; 22:89-95.

Las principales causas de la persistencia del dolor en pacientes con failed back surgery syndrome [FBSS] son fibrosis epidural, hernia de disco y estenosis. La epiduroscopia tiene buenos resultados a largo plazo en aproximadamente el $40 \%$ de los pacientes con FBSS grave que no han respondido a otros tratamientos convencionales como la radiofrecuencia o la epidurolisis con catéter Racz $[1,2]$.

Martin Avellanal y cols. (3) presentan en su artículo un abordaje nuevo para poder realizar el tratamiento cuando los pacientes están afectados de un problema más a nivel foraminal que a nivel central, todos ellos sometidos a una intervención quirúrgica, lo que el Dr. Rafaelli denominó inestabilidad funcional (4), realizando un abordaje transforaminal como alternativa al clásico por vía caudal o al desarrollado por el mismo grupo en el abordaje interlaminar.

En todos los pacientes la adhesiólisis percutánea y la radiofrecuencia habían fallado, por lo que se plantearon la realización de una endoscopia espinal con el objetivo de mejorar la condición de un porcentaje significativo de pacientes antes de ser sometidos a estimulación de la médula espinal u otros tratamientos paliativos.

Se trata de un abordaje menos invasivo y con una tasa baja de complicaciones menores. El menor volumen utilizado, en comparación con el abordaje caudal, entre seis y doce veces menor, según los artículos [5], disminuye la posibilidad de complicaciones neurológicas asociadas al incremento de la presión intracanal. En su experiencia, las adherencias causantes de dolor radicular se ubicaron en los primeros 1 a $2 \mathrm{~cm}$ desde el foramen, lo que facilita el acceso a nivel del receso lateral para su tratamiento. Por tanto esta vía de abordaje parece, según la descripción de los autores, más sencilla y directa. La incidencia de cefalea postpunción dural es nula en relación con lo publicado con la epidurolisis y con el abordaje interlaminar (21\%) en otra serie de casos [6].

Lo segundo que llama la atención son los buenos resultados que se obtienen sin emplear ninguna herramienta que pueda eliminar o pueda cauterizar la fibrosis; es interesante que pacientes sometidos a tratamientos transforaminales no respondan a este tipo de tratamientos y lo hagan a largo plazo y con una disminución tan importante del dolor sin complicaciones.

Para estos pacientes, la epiduroscopia transforaminal mostró resultados significativamente mejores a largo plazo (el $54 \%$ de los pacientes obtienen una reducción del dolor > 50 \%) en comparación con otros enfoques convencionales. La endoscopia transforaminal también se puede emplear como técnica consecutiva en el mismo procedimiento cuando no se puede alcanzar el receso epidural de dentro a fuera con el abordaje caudal. La epiduroscopia parece ir ganando indicaciones y nuevas modalidades de utilización, que junto a los avances técnicos pueden mejorar la efectividad en la difícil patología axial. De todas formas, en necesario aumentar la casuística y los estudios controlados. 


\section{BIBLIOGRAFÍA}

1. Vanelderen P, Richardson J, Van Zundert J, Heavner J, Groen GJ. Epiduroscopy for patients with lumbosacral radicular pain. Pain Pract 2014;14(4):365-77. DOl: 10.1111/papr.12104.

2. Avellanal M, Diaz-Reganon G, Orts A, Soto S. One-year results of an algorithmic approach for managing failed back surgery syndrome. Pain Res Manag 2014;19(6):313-6.

3. Avellanal M, Diaz-Reganon G, Orts A, Gonzalez-Montero L, Riquelme I. Transforaminal Epiduroscopy in Patients with Failed Back Surgery Syndrome. Pain Physician 2019;22(1):89-95.
4. Raffaeli W, Righetti D. Surgical radiofrequency epiduroscopy technique (RResAblator) and FBSS treatment: Preliminary evaluations. Acta Neurochir Suppl 2005;92:121-5.

5. Hwang B-Y, Ko H-S, Suh J-H, Shin J-W, Lee, J-G, Lee J-D. Clinical experiences of performing transforaminal balloon adhesiolysis in patients with failed back surgery syndrome: Two cases report. Korean J Anesthesiol 2014;66(2):16972. DOI: 10.4097/kjae.2014.66.2.169.

6. Avellanal M, Diaz-Reganon G. Interlaminar approach for epiduroscopy in patients with failed back surgery sindrome. British Journal of Anaesthesia 2008;101(2):244-9. DOI: 10.1093/bja/aen165. 